\title{
Correction of the Effect of Particle Shape on the Size Distribution Measured with a Laser Diffraction Instrument
}

\author{
Camiel M. G. Heffels, Peter J. T. Verheijen, Daniel Heitzmann, Brian Scarlett*
}

(Received: 4 October 1995)

\begin{abstract}
This paper gives a theoretical basis for calculating Fraunhofer diffraction patterns of arbitrary polyhedron particles. It is shown that this solution can be used for calculating a scatter matrix adapted to the particle shape in a straightforward manner. Some simulations were made to show the difference
\end{abstract}

between the size distribution by volume obtained with a scatter matrix for spheres and with that for the appropriate shape. Finally, some experimentally measured signatures from platelets and rods are evaluated in order to show that the spherical equivalent diameter could be accurately retrieved.

\section{Introduction}

Many researchers have seen peculiar effects in the particle size distribution when measuring platelets or elongated particles with commercial laser diffraction instruments [1]. Particle shapes which differ strongly from a spherical shape cause the appearance of tails in the volume distribution, either in the small particle size classes (platelets or tablets) or in the large particle size classes (elongated shapes). These effects are the result of the assumption of spherical particles which is currently employed by all the instrument manufacturers.

Diffraction theory for arbitrarily sharp-edged particles allows the calculation of scatter matrices which are specially adapted to particles of a polyhedron shape. It was shown that particle shape can have a much stronger effect on the radial intensity profiles than the refractive index [2]. This paper presents both a theoretical and an experimental study to show that the diameter of the equivalent circle by area can be obtained by a commercial laser diffraction instrument if the particle shape is taken into account in the calculation of the scatter matrix. The equivalent spherical diameter by volume can, of course, also be determined. Since diffraction particle sizing is based on the projected particle area, the equivalent circle diameter of differently shaped particles was determined by a commercial laser diffraction instrument.

\section{Calculating the Fraunhofer Diffraction Matrix}

The Fraunhofer solution for forward light scattering from arbitrarily shaped objects is a straightforward solution for calculating a scatter matrix. The solution is especially useful,

* Dr. C. M. G. Heffels, P. J. T. Verheijen, D. Heitzmann, Prof. B. Scarlett, Delft University of Technology, Faculty of Mechanical Engineering and Marine Technology, Laboratory for Process Equipment, Leeghwaterstraatt 44, NL-2628 CA Delft (The Netherlands). because it is invariant in the quantity $\rho=\alpha \sin \theta$, where $\alpha=\pi x / \lambda$ is the dimensionless particle size and $\theta$ is the radial scatter angle. The quantity $x$ is the equivalent circle area diameter. This invariance is not valid for the anomalous diffraction solution and for the general Lorenz-Mie solution, because these calculations are strongly influenced by particle size-dependent phase effects of light passing through the particle [2]. The restrictions of diffraction theory can be summarized as follows:

- $r>>x$

- $\alpha<r / x$,

- the light beam must have a homogeneous intensity over the illuminated particle area,

where $r$ is the observation distance. The Fraunhofer solution for an arbitrary polygonal particle contour will be derived below in an easier form than in Reference [2]. The Kirchhoff diffraction integral [3]

$\psi^{F D}(\theta, \varphi)=\frac{\psi_{0}}{\lambda R} \iint_{S} \mathrm{e}^{j k A x} \mathrm{e}^{j k B y} d x d y$

$A=\cos \phi \sin \theta$

$B=\sin \phi \sin \theta$

describes the complex diffraction amplitude in the far-field approximation. Eq. (1) is known as the scalar diffraction approximation, which does not take polarization effects into account. The wavenumber is given by $k=2 \pi / \lambda$, where $\lambda$ is the wavelength of the light. $\psi_{0}$ is the uniform complex amplitude in the aperture. The superscript FD means Fraunhofer diffraction. The integration is carried out over the polygonal surface $S$ forming the projected particle shape with the additional requirement $\left\{\left(\mathrm{x}_{\mathrm{i}}, y_{\mathrm{j}}\right) ; \mathrm{i}=1, \ldots, \mathrm{m}\right\}$. The symbols are explained in Figure 1. 


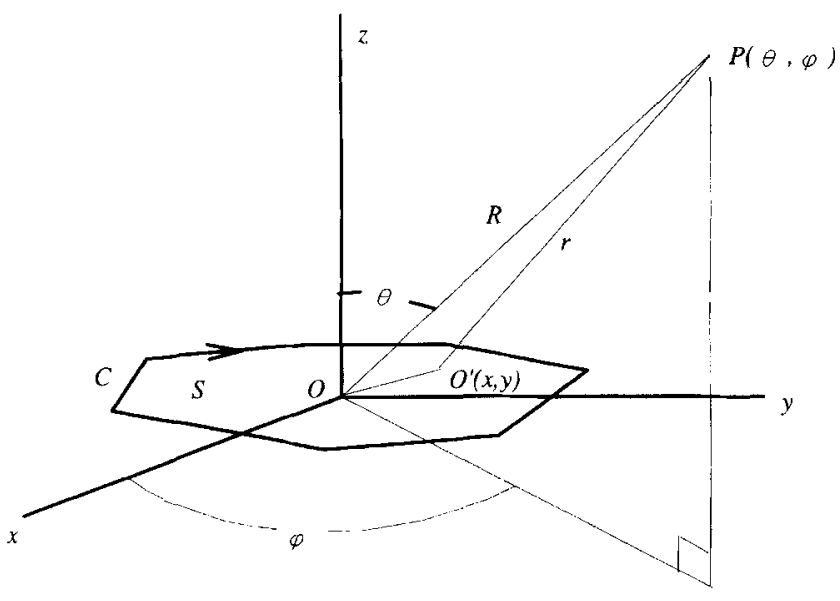

Fig. 1: Geometrics involved in calculating the diffraction integral from a polygonal object.

The two-dimensional integral is transformed to a contour integral with a corollary of Green's theorem [ ]:

$\iint_{S}\left(\frac{\partial^{2} P}{\partial x^{2}}+\frac{\partial^{2} P}{\partial y^{2}}\right) d x d y=\int_{C} \frac{d P}{d n} d s$.

The function $\psi^{F D}(\theta, \varphi)$ is obtained with

$P(x, y)=\frac{-\psi_{0}}{k^{2} \lambda R \sin ^{2} \theta} \mathrm{e}^{j k[A x+B y]}$,

substituted in the left-hand side of Eq. (2). The right hand side of Eq. (2) provides a way of evaluating the integral over each side of the polygon, which is most easily done in the $x^{\prime}, y^{\prime}$-axis system:

$P(x, y)=\frac{-\psi_{0}}{k^{2} \lambda R \sin ^{2} \theta} \mathrm{e}^{j k\left[A^{\prime} x^{\prime}+B^{\prime} y^{\prime}\right]}$,

with

$A^{\prime}=A \cos \gamma+B \sin \gamma \quad x^{\prime}=x \cos \gamma+y \sin \gamma$

$B^{\prime}=-A \sin \gamma+B \cos \gamma \quad y^{\prime}=-x \sin \gamma+y \cos \gamma$,

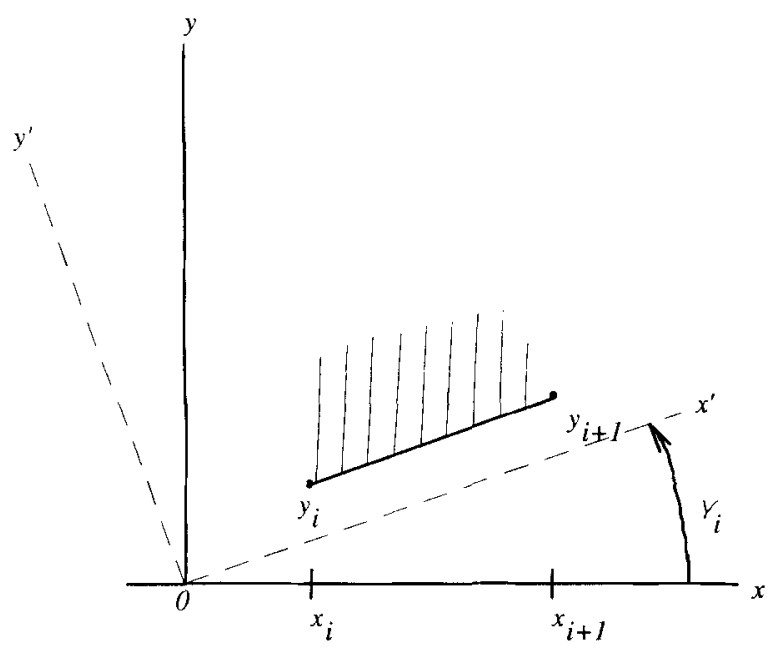

Fig. 2: Graphical explanation of the reference axis rotation for a single line piece. where

$\tan \gamma_{i}=\frac{y_{i+1}-y_{i}}{x_{i+1}-x_{i}}$

The rotation angle $\gamma_{i}$ of the coordinate transformation is shown graphically in Figure 2.

For $A^{\prime} \neq 0$ the line integral was evaluated:

$$
\begin{aligned}
\int_{x_{i}}^{x_{i}+1} \frac{d P}{d n} d s= & \int_{x_{i}^{\prime}}^{x_{i+1}^{\prime}}-\frac{\partial P}{\partial y^{\prime}} d x^{\prime} \\
= & \frac{\psi_{0}}{k^{2} \lambda R \sin ^{2} \theta} \frac{j k B^{\prime}}{j k A^{\prime}}\left(\mathrm{e}^{j k A^{\prime} x_{i+1}^{\prime}}-\mathrm{e}^{j k A^{\prime} x_{i}^{\prime}}\right) \\
& \times\left\{\begin{array}{c}
\mathrm{e}^{j k B^{\prime} y_{i+1}^{\prime}} \\
\text { or } \\
\mathrm{e}^{j k B^{\prime} y_{i}^{\prime}}
\end{array}\right\} \\
= & \frac{\psi_{0}}{k^{2} \lambda R \sin ^{2} \theta} \frac{-A \sin \beta_{i}+B \cos \beta_{i}}{A \cos \beta_{i}+B \sin \beta_{i}} . \\
& \left(\mathrm{e}^{\left.j k\left[A^{\prime} x_{i+1}^{\prime}+B^{\prime} y_{i+1}^{\prime}\right]-\mathrm{e}^{j k\left[A^{\prime} x_{i}^{\prime}+B^{\prime} y_{i}^{\prime}\right]}\right) .}\right.
\end{aligned}
$$

However, when $A^{\prime}=0$ or $\varphi=\gamma_{i} \pm \pi / 2$, the line integral reads

$$
\begin{aligned}
\int_{x_{i}}^{x_{i+1}} \frac{d P}{d n} d s & =\int_{x_{i}^{\prime}}^{x_{i+1}^{\prime}}-\frac{\partial P}{\partial y^{\prime}} d x^{\prime} \\
& =\frac{\psi_{0}}{k^{2} \lambda R \sin ^{2} \theta} j k B^{\prime}\left(x_{i+1}^{\prime}-x_{i}^{\prime}\right) \cdot\left\{\begin{array}{c}
\mathrm{e}^{j k B^{\prime} y_{i+1}^{\prime}} \\
\text { or } \\
\mathrm{e}^{j k B^{\prime} y_{i}^{\prime}}
\end{array}\right\} .
\end{aligned}
$$

The diffraction integral of a polygon can now be written as the sum of line integrals over the line pieces between the corner points $i=1, \ldots, \mathrm{m}$. For $A^{\prime} \neq 0$ this reduces to

$$
\begin{aligned}
\psi_{0}^{F D}(\theta, \varphi)= & \frac{\psi_{0}}{k^{2} \lambda R \sin ^{2} \theta} \sum_{i=1}^{m} \frac{\sin \left(\varphi-\gamma_{i}\right)}{\cos \left(\varphi-\gamma_{i}\right)} \\
& \times\left(\mathrm{e}^{j \hbar\left\lfloor A x_{i+1}+B y_{i+1}\right]}-\mathrm{e}^{\left.j k \mid A x_{i}+B y_{i}\right]}\right) .
\end{aligned}
$$

The intensity distribution needed for obtaining the detector signals is calculated by the expression

$I(\theta, \varphi)=\left|\psi_{0}^{F D}(\theta, \varphi)\right|^{2}$

Since the calculation of a scatter matrix involves an integration of the intensity distribution over the surface of the photodiodes (scatter angles $\theta$ and $\varphi$ ) and an integration over the particle size classes, it would be very time consuming to make the calculation for arbitrarily oriented particles. Only in the Fraunhofer approximation can another approach be taken. First the radial scatter profile was calculated, with Eq. (8), as a function of $\rho$, integrated over a sufficient number of 
three-dimensional particle orientations. Second, this profile was fitted with a function which can be integrated twice over an interval fixed by the detector radii and the size class boundaries. Of course, numerical integration can also be applied but is less efficient in terms of computing time. In this work a polynomial fit was chosen to approximate the radial Fraunhofer scatter profile (averaged over $\varphi$ ) from randomly oriented particles. The terms of the polynomial were integrated individually to obtain the scatter matrix elements. This procedure is illustrated for the case of spherical particles. The equation for a polynomial, fitted to the radial scatter profile of spheres, is expressed, in the Fraunhofer approximation, by

$y(\rho)=\left[\frac{2 J_{1}(\rho)}{\rho}\right]^{2}=\sum_{i=0}^{n} a_{i} \rho^{i}$.

This expression, including the Bessel function, is known as the Airy function. It has the value 1 for $\rho=0$. Eq. (10) does not include the particle size dependence of the absolute scattered intensity. In fact, the constraint $a_{0}=1$ can be made for $\rho=0$, but the angle $\theta=0^{\circ}$ falls out of the integration range, because of the central hole in the detector. Therefore, all the coefficients were varied to allow a best possible fit on the whole interval of $\rho$. The important instrumental quantities $r$ (detector radial coordinate from the central hole) and $f$ (the focal length of the Fourier lens) are related by

$\rho=\frac{\pi}{\lambda f} x \cdot r=\beta \cdot x \cdot r$.

The particle size-dependent radial intensity profile is then described by

$I(\rho) \cong \beta^{2} x^{4} \sum_{i=0}^{n} a_{i} \rho^{i}$

The detector ring signal for a particular size follows from a first integration:

$$
\begin{aligned}
I_{\Delta r_{k}}(x) & =\int_{r_{i \text { iner }}}^{r_{k}^{\text {outer }}} \ln (\rho) r d r \\
& =x^{2} \int\left[\sum_{i=0}^{n} a_{i} \rho^{i+1}\right] d \rho \\
& =x^{2} \sum_{i=0}^{n} \frac{a_{i}}{i+2}\left[\left(\beta x r_{k}^{\text {outer }}\right)^{i+2}-\left(\beta x r_{k}^{\text {inner }}\right)^{i+2}\right] .
\end{aligned}
$$

The scatter matrix elements result from a second integration over the size intervals. An additional factor was included in the integration of particle size in order to calculate the scatter matrix on a volume basis. Therefore, the number-based size distribution function $q_{0}(x)$ was related to the volume-based size distribution function $q_{3}(x)$ by the relationship

$q_{0}(x)=\frac{q_{3}(x)}{x^{3}}$
It is assumed that the size class intervals are narrow enough to substitute the function value of $q_{3}(x)$ in the size class by $q_{3}\left(\bar{x}_{l}\right)$, where $\bar{x}_{l}$ is the arithmetic mean size of the class. The volume-based matrix elements were then calculated with

$$
\begin{aligned}
& M(k, l)=\int_{x_{l}^{\text {power }}}^{x_{l}^{\text {upper }}} \frac{1}{x^{3}} I_{\Delta r_{k}}(x) d x \\
& =\sum_{i=0}^{n} \frac{a_{i}}{i+2} \int \frac{1}{x}\left[\left(\beta x r_{k}^{\text {outer }}\right)^{i+2}-\left(\beta x r_{k}^{\text {inner }}\right)^{i+2}\right] d x \\
& =\sum_{i=0}^{n} \frac{a_{i}}{i+2}\left[\begin{array}{ccc}
\beta r_{k}^{\text {outer }} & \text { upper } & \beta r_{k}^{\text {inner }} \\
x_{l}^{\text {upper }} \\
\int y^{i+1} d y-\int y^{i+1} d y \\
\beta r_{k}^{\text {outer }} x_{l}^{\text {lower }} & \beta r_{k}^{\text {inner }} x_{l}^{\text {lower }}
\end{array}\right] \\
& =\sum_{i=0}^{n} \frac{a_{i}}{i+2}\left[\left(\beta r_{k}^{\text {outer }} x_{l}^{\text {upper }}\right)^{2}-\left(\beta r_{k}^{\text {outer }} x_{l}^{\text {lower }}\right)^{2}\right. \\
& \left.-\left(\beta r_{k}^{\text {inner }} x_{l}^{\text {upper }}\right)^{2}+\left(\beta r_{k}^{\text {inner }} x_{l}^{\text {lower }}\right)^{2}\right] \text {. }
\end{aligned}
$$

The size class boundaries were chosen according to

$x_{l} \cdot r_{k}-\frac{y_{m} \lambda f}{\pi}$,

where $y_{m}=1.357$ is the maximum value of the function $J_{1}^{2}$ $(y) / y$, which describes the average radial scatter intensity of a sphere. Eq. (16) reveals a size distribution vector containing as many elements as available ring signals, which is 31 for the instrument used in the experiments.

The scatter matrices were calculated for the specific shape of the tailor-made particles which were used in the experiments and additionally for tetrahedrons. The particles were made of iron and copper to ensure that they were fully opaque and that the Fraunhofer model was applicable. The polynomial fit was calculated with the POLYFIT algorithm of the software package MATLAB version 4.2c. The shape of all the tailormade particles was approximated by cuboids, including the copper rods. The specific dimensions are given in Table 1 together with an illustration.

The equivalent circle diameter by area, $x$, of these particles is calculated with Cauchy's theorem, which states that the mean projected area of a convex particle is a quarter of its surface area. Table 1 also gives the circular equivalent area diameter, $x$, of the measured particles.

The radial scatter profile was a calculated average over 125 random orientations of the cuboid. The polynomial expansion was fitted with 42 terms. Fewer terms produce worse fits and more terms give heavy oscillations for large values of $p$, probably owing to limited number accuracy in the computer. The residuals of the polynomial fits were calculated with

residual of fit $=\frac{\sum_{i=1}^{\#}\left(y_{i}-y_{i} \text { polyfit }\right)^{2}}{\#-n}$,

where $\#=153$, the number of fitted points, which is larger than the degree of the polynomial $(n=42)$. The residual was at a minimum value when the polynomial contained 42 terms. These minimum values of the residual are tabulated in Table 2. The quality of the fits for the different particle shapes, including the fitted profile for a sphere, is demonstrated in Figure 3. A 

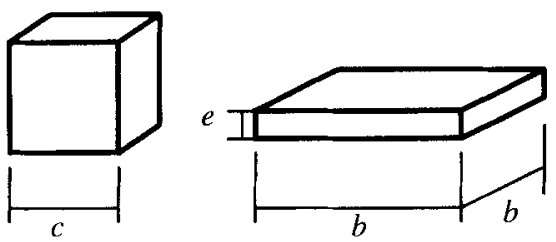

cube

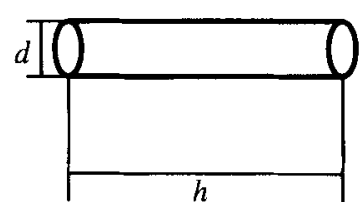

cylinder

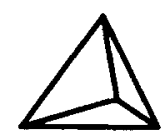

tetrahedron

Table 1: The size of the tailor-made metal particles (see diagrams). All dimensions are in $\mu \mathrm{m}$.

\begin{tabular}{lccr} 
& Iron cubes & Stainless-steel square tablets & Cylindrical copper \\
\hline Dimensions & $c=500$ & $e=150$ & $d=40$ \\
Dimensions & & $b=700$ & $h=250$ \\
Circular equiv. & $x=691$ & $x=668$ & $x=104$
\end{tabular}

area diameter

Table 2: The residual of the polynomial fits up to $\rho=19$, calculated with Eq. (17).

\begin{tabular}{|c|c|c|c|c|c|}
\hline & Spheres & Cubes & $\begin{array}{c}\text { Square } \\
\text { tablets }\end{array}$ & $\begin{array}{l}\text { Square } \\
\text { cuboids }\end{array}$ & Tetrahedra \\
\hline $\begin{array}{l}\text { Residual of } \\
\text { polynom. fit }\end{array}$ & $1.6 \cdot 10^{-10}$ & $6.36 \cdot 10^{-10}$ & $5.18 \cdot 10^{-9}$ & $8.77 \cdot 10^{-6}$ & $1.27 \cdot 10^{-7}$ \\
\hline
\end{tabular}

(a) scatter intensity

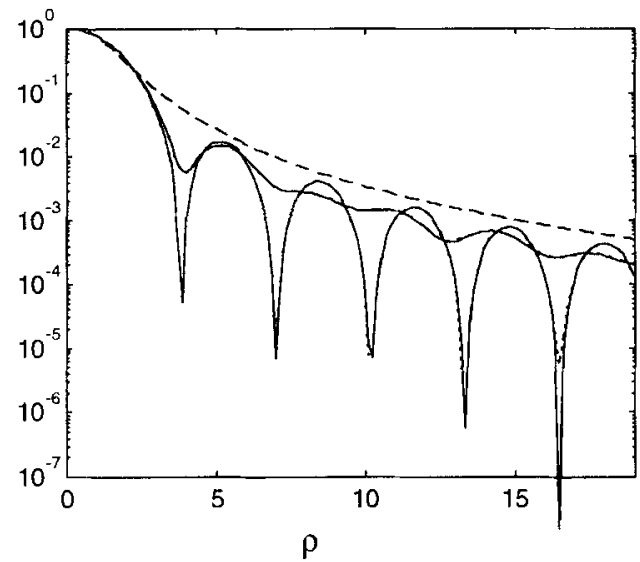

(c) scatter intensity

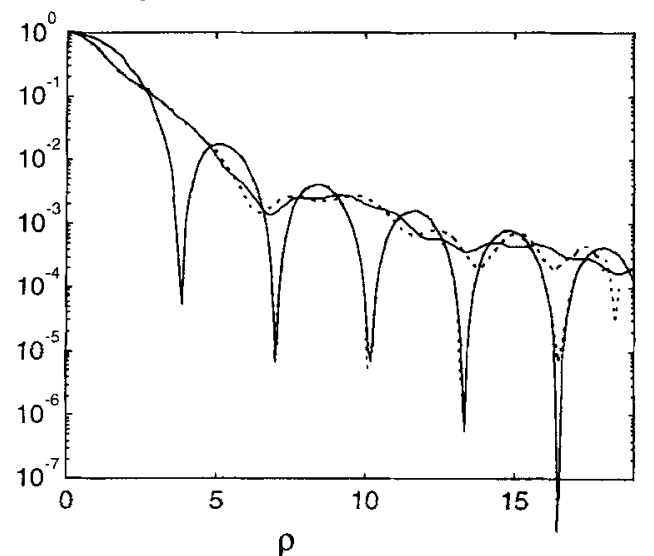

(b) scatter intensity

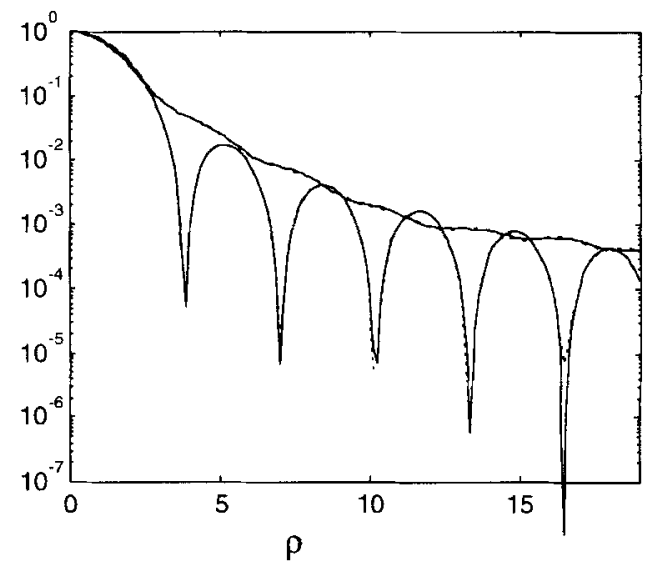

(d) scatter intensity

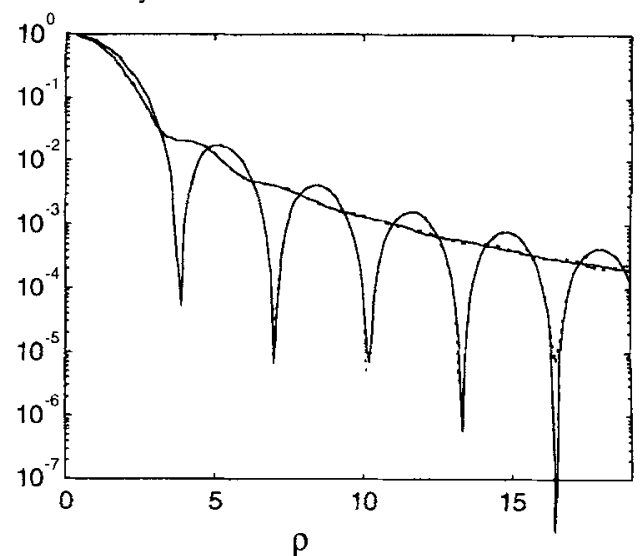

Fig, 3: Fraunhofer scatter profiles of randomly oriented particles; all figures contain the profile for spheres (clear fringe structure). The dotted lines represent the results of the polynomial fits. (a) Cubes, the dashed line is the asymptotic behaviour expressed by Eq. (18); (b) square tablets; (c) square cuboids of aspect ratio 5; (d) tetrahedra. 
(a)

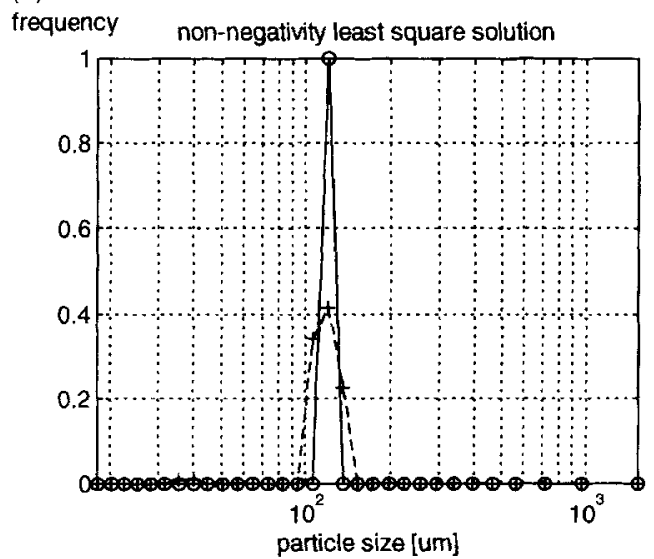

(b)

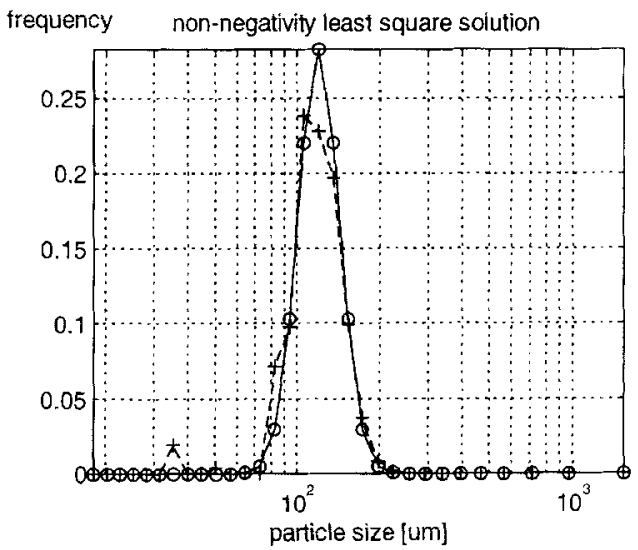

Fig. 4: Calculated particle volume distributions. Dashed line, cube signal inverted with scatter matrix for spheres; solid line, signal inverted with scatter matrix for cubes. (a) Mono size; (b) size distribution (see text).

logarithmic intensity scale was used. The Fraunhofer profiles where calculated at intervals of $\Delta \rho=0.125$.

The polynomial fit began to oscillate heavily for values of $\rho>19$. The scatter intensity was set to 0 for all $\rho>19$. This leads to a discontinuity in the columns of the scatter matrix for the elements belonging to the largest size classes and the large ring detectors. Better fits can probably be made with, for example, a Fourier expansion instead of a Taylor expansion. A Fourier expansion can also be integrated twice, but does not have the implicit asymptotic behaviour for $\rho \rightarrow 0$. The expansion

$I(\rho)=\beta^{2} x^{4} \frac{1}{1+0.29 \cdot \rho^{3}} \sum_{i=0}^{n} a_{i} \cos \left(b_{i} \rho\right)+c_{i} \sin \left(d_{i} \rho\right)$

will be more suitable, but needs numerical integration to obtain the scatter matrix elements.

\section{Simulated Results from the Particle Size Inversion Step}

The scatter matrices were calculated using the dimensions of the Malvern 2600 semi-ring photo detector [4]. The particle distribution by volume of the artificial particles were simulated both by using the scatter matrix for spheres and by using the scatter matrix for the appropriate shape, see Figures 4-7. The inverted scatter signal was taken from one column of the scatter matrix for the appropriate shape. The inversion of the signal with the scatter matrix corrected for shape gives, therefore, a single sharp peak in the distribution. The inversion of the scatter matrices was achieved with the non-negativity leastsquares method [5], since this method gives the best results when no measurement noise is present. Each of Figures 4-7 shows an inversion of the signal from one size class in the middle size range $\left(x_{\text {middle }}=119.6 \mu \mathrm{m}\right)$ and also an inversion of a log-normal distribution of particles centred around $119.6 \mu \mathrm{m}$ with a width of $124.1 \mu \mathrm{m}$ at $1 / e$ of the peak height.

The scatter matrices for cubes and for spheres do not produce very different particle size distributions compared with the tablets and the square cuboids of aspect ratio 5. The signals from the square tablets, inverted with the matrix for spherical particles, shows a distribution which has a noisy tail in the smaller size classes and the modal value is higher than the equivalent circle diameter by area. From all the calculations it can be concluded that the scatter matrix for spheres does not give a modal value matching the equivalent circle diameter, either for monosized particles or for a size distribution. The distributions resulting from the matrix for spherical particles look noisy. The non-negativity least-squares method gave a larger residual in the case when the scatter matrix for spheres was used, compared with the case of the matrix for the appropriate shape. The noise indicates the erroneous scatter model. The residual of the inversion has been defined as

residual $=(M q-L)^{T}(M q-L)$

The vector $L$ is the light energy signal that is deconvoluted. The residuals belonging to the distributions shown in Figures 4-7 are tabulated, see Table 3 . It must be noted that this definition of the residual is not normalized with the signal level of the light energies. The values in Table 3 therefore cannot be compared directly with the values for the actual measurements which are presented in Table 4.

It is interesting to see that the residual obtained with the matrix for spheres is systematically lower for the size distribution (last row) compared to the mono size case (second row). The smoother profiles can thus be fitted more accurately when the scatter model for spheres is applied.

\section{Experimental Results and Discussion}

The measurements were made with a Malvern 2600 C laser particle sizer equipped with an $f=1000 \mathrm{~mm}$ Fourier lens. The instrument was chosen for its semi-ring detector design which averages out the azimuthal structure in the diffraction pattern even when the particles have a preferential orientation. The sample of tailor-made iron cubes was made with a laser cutter. The copper fibres were hand-made by cutting a thin copper wire. The light energy values on the detector of the tailor made steel tablets were taken from the paper by Gabas et al. [1]. They also used a Malvern 2600 instrument. The detector light energy values are shown graphically in Figure 8 . The values have been corrected with a background measurement. The signals from the cubes and the tablets show an increasing level on the outer rings. This is due either to small particles present in the measurement cell or to changes in the background signals. The 
(a)

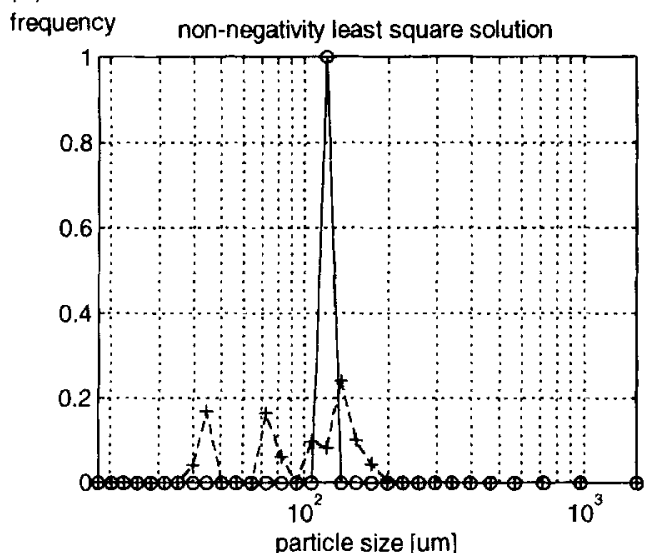

(b)

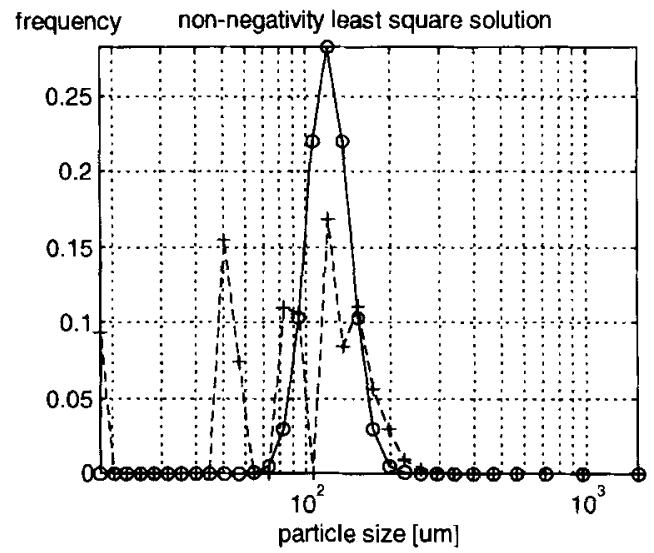

Fig. 5: Calculated particle volume distributions. Dashed line, signal of square tablet inverted with scatter matrix for spheres; solid line, signal inverted with scatter matrix for square tablets. (a) Mono size; (b) size distribution (see text).

(a)

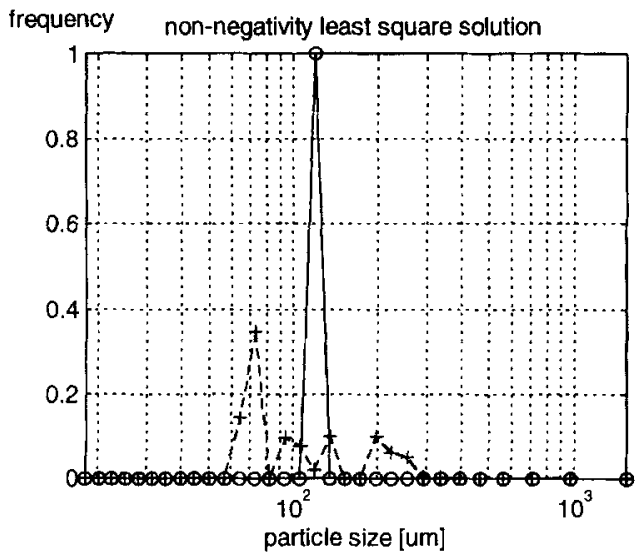

(b)

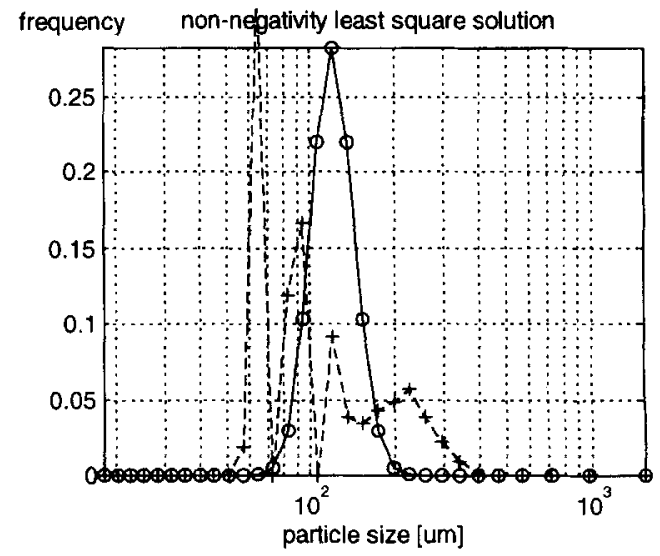

Fig. 6: Calculated particle volume distributions. Dashed line, signal from square cuboid with aspect ratio 5 inverted with scatter matrix for spheres; solid line, signal inverted with a scatter matrix for square cuboids. (a) Mono size; (b) size distribution (see text).

(a)

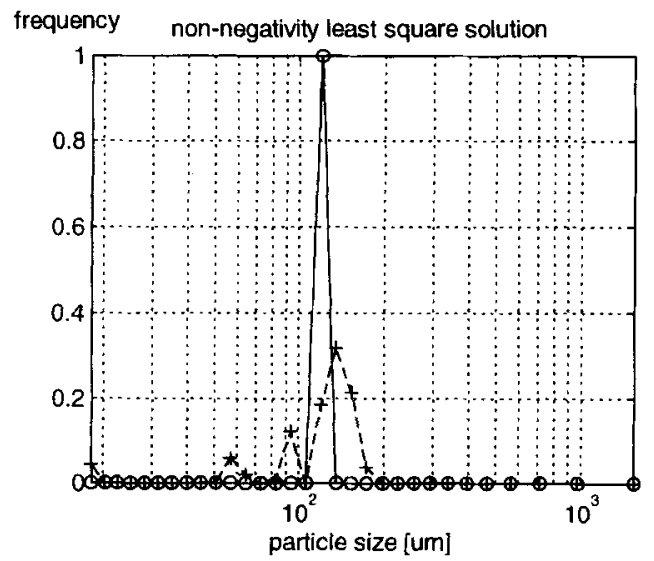

(b)

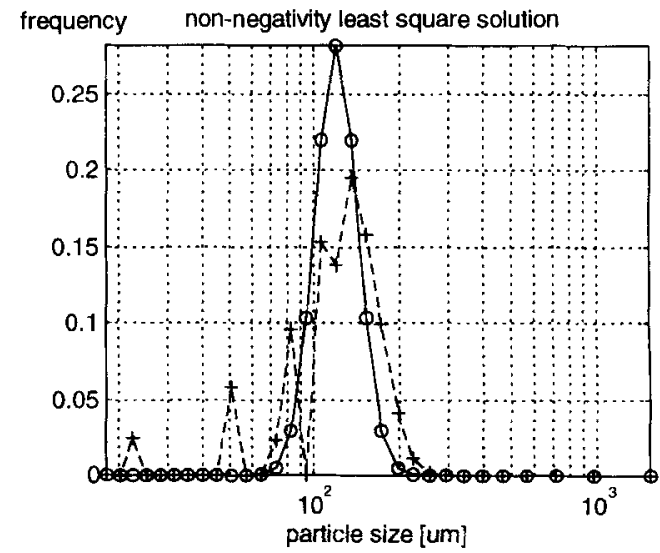

Fig. 7: Calculated particle volume distributions. Dashed line, tetrahedron signal inverted with scatter matrix for spheres; solid line, signal inverted with scatter matrix for tetrahedra. (a) Mono size; (b) size distribution (see text).

Table 3: The residuals of the inversion, for the calculations presented in Figures 4-7.

\begin{tabular}{lllll}
\hline Distribution type: scatter matrix & Cubes & Tablets & Cuboids & Tetrahedra \\
\hline Mono size: matrix for right shape & $1.86 \cdot 10^{-17}$ & $1.04 \cdot 10^{-17}$ & $5.80 \cdot 10^{-18}$ & $8.10 \cdot 10^{-18}$ \\
Mono size: matrix for spheres & $3.99 \cdot 10^{-4}$ & $1.01 \cdot 10^{-5}$ & $7.79 \cdot 10^{-4}$ & $1.87 \cdot 10^{-5}$ \\
Size distribution: matrix for right shape & $8.00 \cdot 10^{-14}$ & $5.18 \cdot 10^{-13}$ & $8.31 \cdot 10^{-10}$ & $5.25 \cdot 10^{-10}$ \\
Size distribution: matrix for spheres & $3.85 \cdot 10^{-7}$ & $1.97 \cdot 10^{-6}$ & $4.72 \cdot 10^{-5}$ & $1.94 \cdot 10^{-6}$ \\
\hline
\end{tabular}


effect appears as additional small particles present in the size distribution, see Figure 8.

The particle volume distributions were calculated with the scatter matrix for spherical particles and with the scatter matrix for the corresponding particle shape. Both the results of the non-negativity least-squares method and the Chahine inversion scheme [4] are presented in Figure 9. The Chahine method was used, because it reacts more smoothly to noise present in the experimental signals.

(a)

\section{background corrected signal [a.u.]}

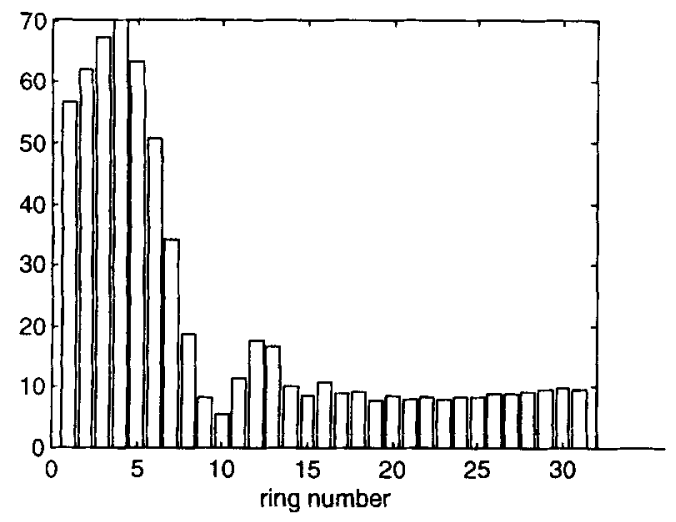

(b)

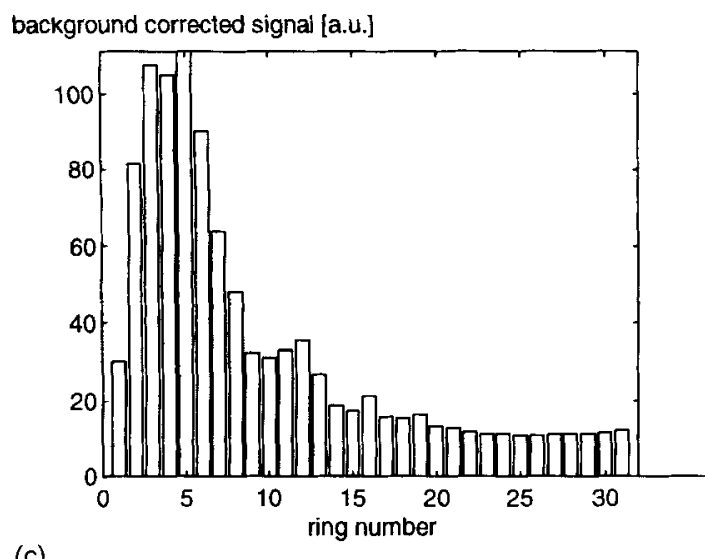

(c)

background corrected signal [a.u.]

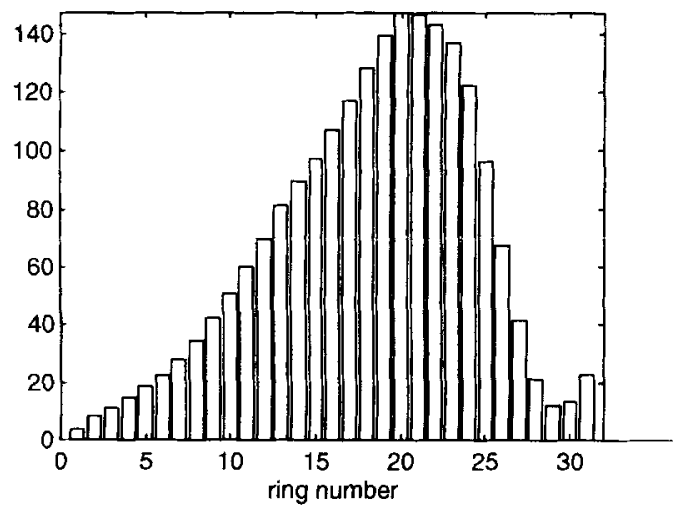

Fig. 8: Malvern 2600 detector signals corrected for background signal. (a) Cubes; (b) square tablets; (c) cylinders of aspect ratio 5.

The theoretical calculations and the size distributions obtained from the experimentally measured signals show identical behaviour. The tailor-made particles produce a narrow peak of the equivalent circle diameter by area, when using the appropriate scatter matrix. The residual of the inversion with the scatter matrix for the correct shape is, however, larger than
Table 4: The residuals of the non-negativity least-squares inversion of the experimental signals.

\begin{tabular}{lccc}
\hline Residuals values of: & Cubes & Square tablets & Rods \\
\hline Matrix for spheres & 7.31 & 42.95 & 11.06 \\
Matrix for right shape & 8.55 & 60.50 & 19.09 \\
\hline
\end{tabular}

the residual obtained with the scatter matrix for spheres, see Table 4.

The reason for the higher residual when using the matrix for the appropriate shape cannot be attributed to a physical model error in this case. At least two causes were identified by Press et al. for the remaining residuals [6]:

- The scattering model used is inadequate. Although the underlying physics of the model is correct, the selected size range and resolution may be inappropriate.

- The random errors in the measurement are, in reality, larger than observed. Probably more readings are required to make a proper estimate of the distribution.

An explanation for the problem of the residual values is that the scatter matrix for cuboidal particles is less well conditioned for inversion [7]. The scatter matrices calculated for elongated particles have a less pronounced structure along the main diagonal. This can be improved to a small extent by taking a more appropriate value for the constant $y_{m}=1.357$ used in calculating the size classes (see Eq. (16)), since this argument belongs to the maximum radial scatter signal for a sphere. However, when the residual of the non-negativity least-squares calculation is plotted against the aspect ratio of the square cuboid used in the matrix inversion, a minimum of the residual was observed at the proper aspect ratio of the measured copper rods, see Figure 10.

Figure 10 reveals that an aspect ratio of 6.5 of the square cuboid is more suitable than the copper rods of aspect ratio 5 , which is not surprising. This observation thus shows the possibility of using the residual as a criterion for selecting the optimum scatter matrix which can be derived from the radial light scattering measurement itself.

\section{Conclusion and Future Developments}

A commercial diffraction particle sizer was used to obtain the spherical equivalent diameter of particles having the approximate shape of a cuboid. To achieve this a scatter matrix which incorporates the shape of the particles was calculated. A generalized Fraunhofer theory for arbitrary sharp-edged particles was used to calculate the scatter matrix. Special attention is needed when the particles have a low refractive index; the Fraunhofer theory cannot be used in that case. The anomalous diffraction theory is an alternative, but the calculation of the scatter matrix is then much more complicated and time consuming.

The difference between the particle size distribution by volume, obtained with a scatter matrix for spherical particles (as used in commercial diffraction instruments), and the distribution obtained with the scatter matrix for the correct particle shape is considered for platelets and elongated particles. For cubes the difference is negligible. The size distribution of copper rods 
(a)

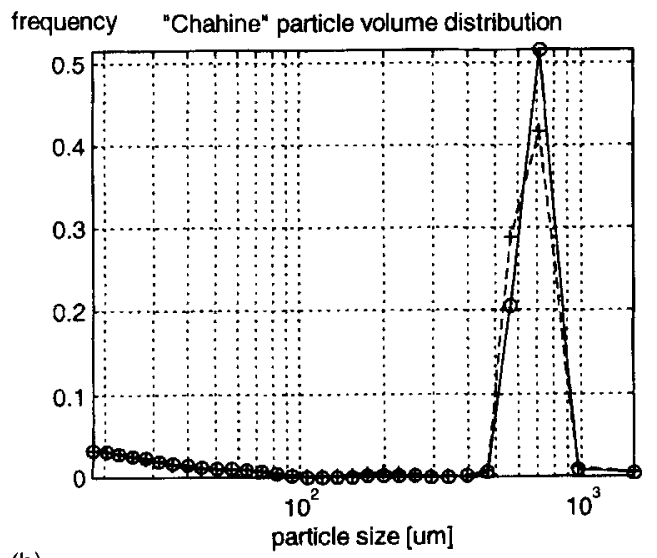

(b)

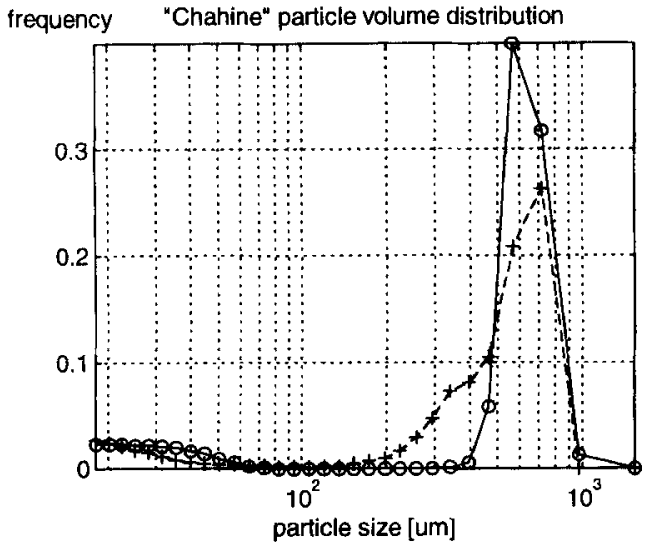

(c)

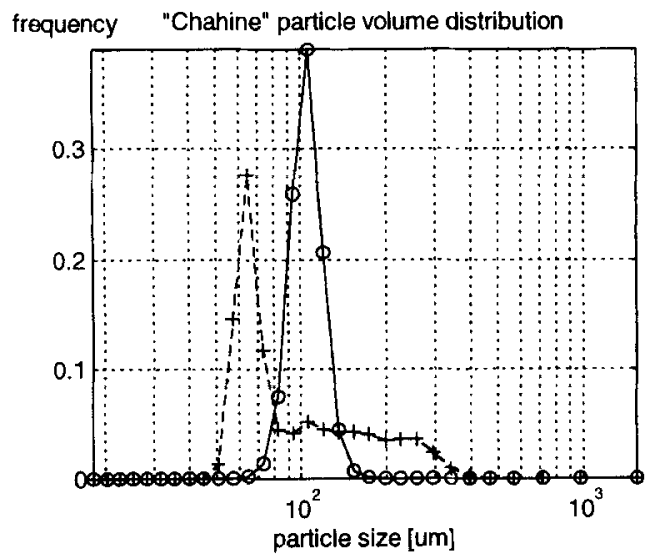

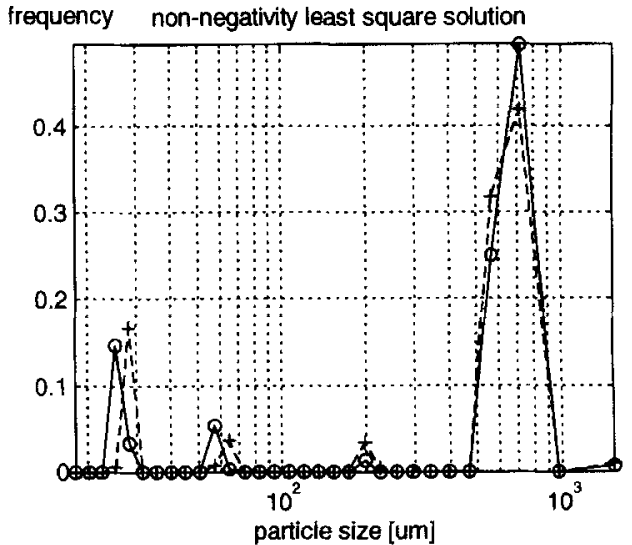
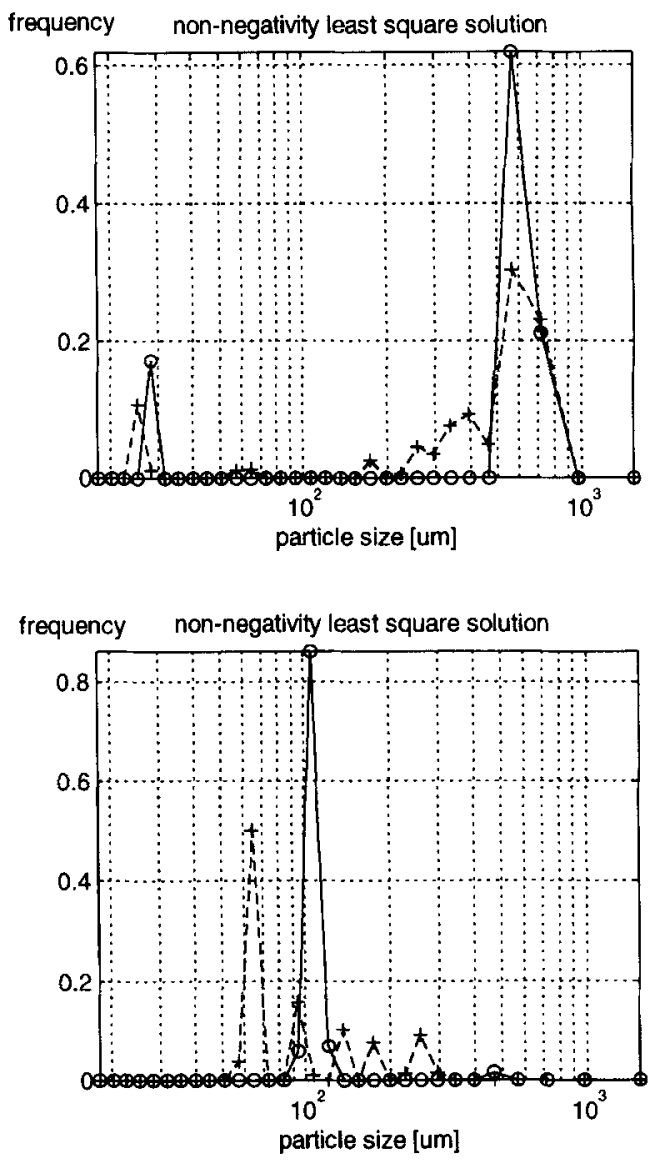

Fig. 9: Particle volume distributions obtained from the measurements of the tailor-made metal particies. The dashed lines present the inversion result with the matrix for spheres and the solid line is the result with the matrix of appropriate shape. (a) Iron cubes; (b) square tablets; (c) copper rods.

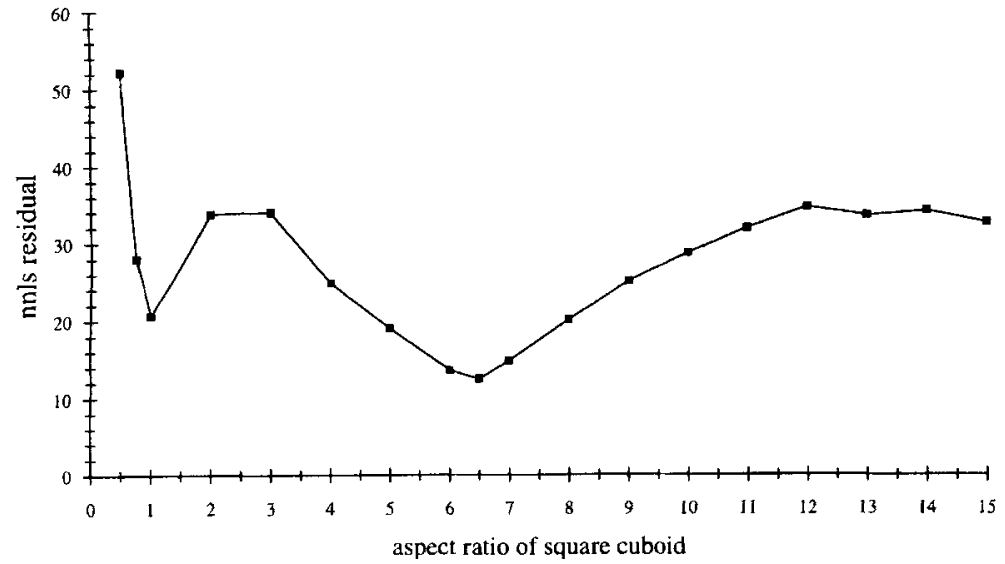

Fig. 10: The residuals of the non-negativity least-squares inversion applied to the copper rod signals as a function of the aspect ratio of the square cuboid used for calculating the scatter matrix 
with an aspect ratio of 5 was successfully obtained by using a scatter matrix calculated for square cuboids of the same aspect ratio. The residual of the particle size inversion step provides a criterion of choosing the optimal scatter matrix.

This work leads to a plausible proposal for selecting the appropriate scatter matrix for a particular measurement from the azimuthal intensity distribution in the diffraction patterns itself. This is a more convenient method than a visual observation of the (average) particle shape before measuring the particle size distribution with the diffraction instrument. The azimuthal intensity fluctuations in diffraction patterns of an ensemble of particles were processed to characterize the average shape of the particles [8]. The selection of the scatter matrix can be based on the azimuthal intensity measurements. This procedure would automate the selection of the appropriate scatter matrix from a database. For this intelligent, self-correcting particle sizer both the radial and azimuthal scatter signatures need to be measured at the same time with the aid of a dedicated detector array [4], which has still to be made.

\section{Symbols and Abbreviations}

$a_{n} \quad$ coefficients of a polynomial

$\alpha \quad$ dimensionless particle size $(=\pi x / \lambda)$

$\beta \quad$ instrumental constant $(=\pi / \lambda f)$

$\gamma \quad$ rotation angle of coordinate system

$\lambda$ wavelength of the light source

$f \quad$ focal length

$k \quad$ wavenumber in vacuum

$L \quad$ measured radial scatter signature

$M \quad$ scatter matrix

$m$ number of cornerpoints of projected particle contour $n$

$q$

$q(x)$

$\rho$

$r_{k}^{\text {outer }}$

$r_{k}^{i n n e r}$

$\theta$

$x$

$x$ upper

$x_{\text {lower }}$ number of terms of the polynomial

size distribution vector

size distribution function

invariant quantity in Fraunhofer solution $(=\alpha \sin \theta)$

outer ring radius of ring $k$

inner ring radius of ring $k$

radial scatter angle

circular equivalent area diameter

upper diameter limit of size class $l$

lower diameter limit of size class $l$

\section{References}

[1] N. Gabas, N. Hiquily, C. Laguérie: Response of Laser Diffraction Particle Sizer to Anisometric Particles. Part. Part. Syst. Charact. 11 (1994) 121-126.

[2] C. Heffels, D. Heitzmann, E. D. Hirleman, B. Scarlett: Forward light scattering from sharp edged-crystals in Fraunhofer and anomalous diffraction approximations. Appl. Opt. 34 (1995) 5.

[3] E. Hecht: Optics, 2nd ed. Addison-Wesley, Reading, MA, 1987, Chap. 10, p. 458 and Appendix 2, p. 623.

[4] C. M. G. Heffels: On-line particle size and shape characterization by small angle light scattering. PhD thesis, Delft University of Technology, Delft University Press, The Netherlands, 1995.

[5] C. L. Lawson, R. J, Hanson: Solving Least Square Problems. Prentice-Hall Englewood Cliffs, NJ 1974. Chap. 23, p. 161.

[6] W. H. Press, B. P. Flannery, S. A. Teukolsky, W. T. Vettering: Numerical Recipes in C: The Art of Scientific Computing. Cambridge University Press, Cambridge 1988.

[7] E. D. Hirleman: Optimal Scaling of the Inverse Fraunhofer Diffraction Particle Sizing Problem: the Linear System Produced by Quadrature. Part Charact. 4 (1987) 128-133.

[8] C. M. G. Heffels, D. Heitzmann, E. D. Hirleman, B. Scarlett: The Use of Azimuthal Intensity Variations in Diffraction Patterns for Particle Shape Characterization. Part. Part. Syst. Charact. 11 (1994) 194-199. 